\title{
Polymorphismes des gènes APOE et ACE dans la longévité humaine
}

Les variations inter-individuelles de la longévité humaine sont en partie dues à une composante génétique, mise en évidence par diverses études familiales [1]. Afin d'identifier les variants génétiques concernés, une banque d'échantillons de centenaires a été constituée au CEPH (Centre d'étude du polymorphisme humain). Nous présentons ici les résultats d'une première étude d'associations génétiques avec la longévité, portant sur plus de 300 centenaires et 200 témoins adultes [2]. Les maladies cardiovasculaires représentant la première cause de décès en France, les premiers gènes candidats étudiés ont été choisis pour l'impact de leurs variants sur le risque cardiovasculaire: il s'agit des gènes de l'apolipoprotéine B (ApoB), l'apolipoprotéine E (ApoE) et l'enzyme de conversion de l'angiotensine (respectivement $A P O B, A P O E$ et $A C E)$.

$A P O E$ possède trois allèles communs : $\varepsilon 2$, $\varepsilon 3$ et $\varepsilon 4$, qui correspondent à trois isoformes distinctes, différant par des mutations ponctuelles [3, 4]. Un polymorphisme biallélique d'insertion-délétion (I/D) dans le gène de l'ACE a été associé à un effet génétique important sur la concentration plasmatique d'ACE [5]. Deux polymorphismes bialléliques dans le gène de l'ApoB ont été sélectionnés pour leur impact sur le taux de cholestérol plasmatique et l'athérosclérose coronarienne [6].

Notre étude a révélé des associations de la longévité humaine avec les gènes $A P O E$ et $A C E$ mais pas avec $A P O B$. La fréquence de l'allèle $\varepsilon 4$ de $A P O E$ est diminuée chez les centenaires $(5,2 \%$ contre $11,2 \%$ dans le groupe témoin, $\mathrm{p}<0,001)$, tan- mentée $(12,8 \%$ contre $6,8 \%$, $\mathrm{p}<0,01)$. La fréquence de l'allèle commun $\varepsilon 3$ reste quasiment inchangée. L'allèle $D$ du gène $A C E$ est plus fréquent chez les centenaires (39,6\% d'homozygotes $D D$ contre $25,6 \%$ dans le groupe témoin, $\mathrm{p}<$ $0,01)$.

Les mêmes associations se retrouvent dans un sous-groupe, sélectionné indépendamment par un autre protocole, de centenaires ayant des frères ou sœurs très âgés et vivants (hommes ayant dépassé l'âge de 90 ans, femmes ayant dépassé l'âge de 95 ans). Cela confirme et renforce les effets observés. Par ailleurs, aucune association de la longévité n'a été détectée avec des polymorphismes dans deux autres loci situés sur d'autres chromosomes: le gène BCL2, sur le chromosome 18 , et la région du gène de la maladie de Huntington, sur le chromosome 4. Ces effets comptent parmi les premières influences génétiques sur la longévité décrites chez l'homme, en dehors du locus HLA [7, 8]. Ces trois associations génétiques soulèvent chacune de nouvelles questions. La diminution de la fréquence du variant ApoE4 chez les centenaires est cohérente avec ce que l'on sait de son impact sur le cholestérol plasmatique lié aux LDL (low density lipoproteins) [9], ainsi qu'avec plusieurs études l'impliquant comme facteur de risque dans les cardiopathies ischémiques $[9,10]$. En outre, ce même variant prédispose aux formes tardives, familiales et sporadiques, de la maladie d'Alzheimer, ce qui pourrait encore contribuer à sa disparition aux âges avancés ([11], $\mathrm{m} / \mathrm{s}$ $n^{\circ}$ 10, vol.9, p. 1142). Comme aucune sélection négative ne peut s'exercer sur des effets délétères se manifestant après l'âge de la repro- duction, ce variant illustre bien une théorie désormais classique: en l'absence de pression sélective, des mutations néfastes à la survie de l'organisme pourraient s'accumuler au cours de l'histoire de l'espèce [12]. Cependant, la susceptibilité accrue aux atteintes coronariennes associée à l'allèle $\varepsilon 4$ semble concerner une population masculine assez jeune [10] pour que s'exprime une sélection négative; cet allèle est, néammoins, maintenu à une fréquence stable supérieure à $10 \%$ dans la population. Cette pression est-elle négligeable, ou bien $\varepsilon 4$ confère-t-il certains avantages sélectifs qui restent à découvrir? Quant au variant ApoE2, le principal argument en faveur d'un effet protecteur serait son impact négatif sur le taux de LDL-cholestérol plasmatique [9]. Toutefois, jusqu'à la nôtre, aucune étude de génétique des populations n'avait indiqué un rôle protecteur; au contraire, $\varepsilon 2$ avait été associé à l'hyperlipidémie de type III et même, dans la population australienne, à l'ischémie cardiaque [13] .

Le résultat le plus inattendu concerne la fréquence de l'allèle $A C E / D$ chez les centenaires. En effet, plusieurs études sur des populations françaises ont montré que cet allèle confère un risque accru d'infarctus du myocarde [14, 15] : comment expliquer alors qu'il se retrouve plus fréquemment chez les centenaires? Il convient de noter que le mécanisme qui sous-tend l'augmentation du risque d'infarctus pour les homozygotes $D D$ dans les diverses populations étudiées n'est pas du tout élucidé. L'ACE est une dipeptyl carboxypeptidase qui clive l'angiotensinogène pour donner l'angiotensine I, un vasoconstricteur puissant, et inactive, par clivage, la bradyki- 
nine, un vasodilatateur [16]. Néanmoins, il n'y a pas de corrélation entre l'hypertension et le génotype ACE dans des populations adultes [17]. L'association d' $A C E / D$ avec la longévité pourrait résulter d'effets hors de l'axe rénine-angiotensine et du système cardiovasculaire: l'ACE agit sur d'autres substrats, parmi lesquels des neuropeptides comme l'enképhaline, la LHRH (luteinizing hormone releasing hormone), la neurotensine [18], ou encore des peptides présentés dans le contexte HIA classe I par les cellules accessoires du système immunitaire [19]. L'ACE est exprimée dans le cerveau et les leucocytes et la même corrélation semble exister dans différents tissus entre taux d'expression et génotype, le génotype $\boldsymbol{D} \boldsymbol{D}$ ayant un plus fort taux d'expression que les autres génotypes $D / I$ et $I I[20]$.

Contrairement au cas d' $A P O E$, pour lequel les allèles directement responsables des effets observés sont connus - selon toute probabilité, il s'agit des variants génotypés ceux-ci restent à identifier pour l'ACE. Notons, en passant, la proximité du gène de l'hormone de croissance, dont un rôle dans le vieillissement a fait l'objet de plusieurs travaux [21] : l'association que l'on trouve pourrait résulter d'un déséquilibre de liaison avec un polymorphisme voisin.

Bien qu'elle n'atteigne pas le seuil de signification statistique, il est intéressant de noter une corrélation négative entre les allèles $A P O E 2$ et $A C E / D$ dans la population de centenaires: elle indique une interaction entre les effets du variant $\varepsilon 2$ et du polymorphisme d'ACE dont un mécanisme physiologique devra, en dernière instance, rendre compte. Ces résultats n'appellent pour l'instant que des conjectures, mais ils suggèrent que la longévité résulte de multiples influences génétiques enchevêtrées, couplées à des effets de l'environnement, et intégrées sur toute la durée de l'existence [22].

F.S.

D.C.

L. F.D.
1. Schächter F, Cohen D. Longevity: a new field for human genetics. Lifespan 1993; 4 : 1-3.

2. Schächter F, Faure-Delanef L, Guénot F, Rouger H, Froguel P, Lesueur-(inot L, Cohen D. (jenetic associations with longevity at the $A P O E$ and $A C E$ loci. Nature (jenet 1994 ; $6: 29-32$

3. Zannis VI, Breslow JL, Utermann C; Mahley RW, Weisgraber KH, Havel RJ. Proposed nomenclature of ApoE isoproteins, apoE genotypes, and phenotypes. / Lipid Res 1982 23: 911-4.

4. Cambien F. Polymorphismes génétiques des apolipoprotéines. médecine/sciences 1989; 5 : 379-88.

5. Tiret L, Rigat B, Visvikis S, Breda C, Cor vol P, Cambien F, Soubrier F. Evidence, from combined segregation and linkage analysis, that a variant of the angiotensin l-converting enzyme (ACE) gene controls plasma ACE levels. Am / Hum Genet 1992; 51 : 197-205. 6. Peacock R, Dunning A, Hamsten A, Tornvall $\mathrm{P}$, Humphries S, Talmud P. Apolipoprotein $B$ gene polymorphisms, lipoproteins and coronary atherosclerosis: a study of young myocardial infarction survivors and healthy population-based individuals. Atherosclerosis 1992 ; 92 : 151-64.

7. Proust J, Moulias R, Fumeron F, Bekkhou cha F, Busson M, Schmid M, Hors J. HI A and longevity. Tissue Antigens 1982; 19: 168-73. 8. Takata H, Ishii T, Suzuki M, Sekiguchi S, Iri $\mathrm{H}$. Influence of major histocompatibility complex region genes on human longevity among Okinawan-Japanese centenarians and nonagenarians. Lancet 1987 ; 2 : 824-6.

9. Davignon ], Gregg RE, Sing CF. Apolipoprotein E polymorphism and atherosclerosis Arteriosclerosis 1988; 8: 1-21

10. Van Bockxmeer FM, Mamotte CDS. Apolipoprotein $\varepsilon 4$ homozygosity in young men with coronary heart disease. Lancel 1992 340 : 879-80.

11. Corder EH, Saunders AM, Strittmatter WJ Schmechel DE, Gaskell P(), Small (;W, Roses AD, Haines JL, Pericak-Vance. Gene dose of apolipoprotein $\mathrm{E}$ type 4 allele and the risk of Alzheimer's disease in late onset families. Science 1993; 261 : 921-3.

12. Medawar PB. An unsolved problem of biolog Londres: Lewis, 1952.

13. Eto M, Watanabe K, Makino l. Increased frequencies of apolipoprotein $\varepsilon 2$ and $\varepsilon 4$ alleles in patients with ischemic heart disease. Clin Genet 1989; 36: 183-8.

14. Cambien F, Poirier O, Lecerf L, Evans A Cambou JP, Arveiler D, Luc (;) Bard JM Bara L, Ricard S, Tiret L, Amouyel P, Alhenc Gelas F, Soubrier F. Deletion polymorphism in the gene for angiotensin-converting enzyme is a potent risk factor for myocardial infarction. Nature 1992; 359; 641-4.

15. Tiret L, Poirier O, LecerfL, Cambou JP Arveiler D, Amouyel P, Kee F, Nicaud V, Evans Z, Luc G, Cambien F. Deletion polymorphism in the angiotensin-converting enzyme gene associated with parental history of myocardial infarction. Iancet 1993; 341 : 991-2.

16. Ehlers MRW, Riordan JF. Angiotensinconverting enzyme : new concepts concerning its biological role. Biochemistry 1989; 28 5.311-3.
17. Jeunemaître X, Lifton RP, Hunt SC, Williams RR, Ialouel JM. Absence of linkage between the angiotensin-converting enzyme locus and human essential hypertension. Nature Genet 1992; 1 : 72-5.

18. Erdōs EG. Angiotensin l-converting enzyme and the changes in our concepts through the years. Hypertension 1990; 16 363-70.

19. Eisenlohr LC, Bacik 1, BenninkJR, Bernstein $\mathrm{K}$, Yewdell JW. Expression of a membrane protease enhances presentation of endogenous antigens to MHC class l-restricted $\mathrm{T}$ lymphocytes. Cell 1992; 71 : 963-72.

20. Costerousse $\mathrm{O}$, Allegrini ], Lopez $\mathrm{M}$ Alhenc-Gelas F. Angiotensin l-converting enzyme in human circulating mononuclear cells : genetic polymorphism of expression in T lymphocytes. Biochem / 1993; 290: 33-40. 21. Jorgensen JOL, Christiansen JS. Brave new senescence: $\mathbf{6 H}$ therapy in adults. Lancet $1993 ; 341: 1247$.

22. Schächter F, Cohen D, Kirkwood TBI Prospects for the genetics of human longevity. Hum Genel 1993; 91 : 519-26. 